\title{
A cross-diffusion system derived from a Fokker-Planck equation with partial averaging
}

\author{
Ansgar Jüngel and Nicola Zamponi
}

\begin{abstract}
A cross-diffusion system for two components with a Laplacian structure is analyzed on the multi-dimensional torus. This system, which was recently suggested by P.-L. Lions, is formally derived from a Fokker-Planck equation for the probability density associated with a multi-dimensional Itō process, assuming that the diffusion coefficients depend on partial averages of the probability density with exponential weights. A main feature is that the diffusion matrix of the limiting cross-diffusion system is generally neither symmetric nor positive definite, but its structure allows for the use of entropy methods. The global-in-time existence of positive weak solutions is proved and, under a simplifying assumption, the large-time asymptotics is investigated.
\end{abstract}

Mathematics Subject Classification. 35K45, 35K65, 35Q84.

Keywords. Cross-diffusion system, Fokker-Planck equation, Entropy methods, Global existence of weak solutions, Large-time asymptotics, Positivity of solutions.

\section{Introduction}

The aim of this paper is the analysis of the following cross-diffusion system

$$
\partial_{t} u_{i}=\Delta\left(a\left(u_{1} / u_{2}\right) u_{i}\right)+\mu_{i} u_{i}, \quad t>0, \quad u_{i}(0)=u_{i}^{0} \geq 0 \quad \text { in } \mathbb{T}^{d}, \quad i=1,2,
$$

where $\mathbb{T}^{d}$ is the $d$-dimensional torus with $d \geq 1, a:(0, \infty) \rightarrow(0, \infty)$ is a continuously differentiable function, and $\mu_{i} \in \mathbb{R}$. This system can be formally derived [7] from a $(d+1)$-dimensional Fokker-Planck equation for the probability density $f(x, y, t)$, where $x \in \mathbb{R}^{d}, y \in \mathbb{R}$. The function $u_{i}$ is obtained from $f$ by partial averaging,

$$
u_{i}(x, t)=\int_{\mathbb{R}} f(x, y, t) e^{\lambda_{i} y} d y, \quad i=1,2,
$$

$\mu_{i}$ is a function of $\lambda_{i}$, and $a\left(u_{1} / u_{2}\right)$ is related to the diffusion coefficients in the Fokker-Planck equation. Strictly speaking, Eq. (1) holds in $\mathbb{R}^{d}$ (or on some subset of $\mathbb{R}^{d}$ ), but we consider this equation on the torus for the sake of simplicity (and to avoid possible issues with boundary conditions). For details on the derivation, we refer to Sect. 2 .

System (1) has been suggested by Lions [7], and the global-in-time existence of (weak) solutions has been identified as an open problem. In this paper, we solve this problem by applying the entropy method for diffusive equations.

The underlying Fokker-Planck equation for $f(x, y, t)$ models the time evolution of the value of a financial product in an idealized financial market, depending on various underlying assets or economic values. The function $u_{i}$ is an average with respect to the variable $y$, which may be interpreted as the value

The authors acknowledge partial support from the Austrian Science Fund (FWF), Grants P22108, P24304, and W1245, and from the Austrian-French Project Amadeé of the Austrian Exchange Service (ÖAD), Grant FR 04/2016. 
of an economic parameter, and the exponential weight emphasizes large positive or large negative values of $y$, depending on the sign of $\lambda_{i}$. We note that partial averaging is also employed to simplify chemical master equations [9]. Here, we are not interested in potential applications, but more in the refinement of mathematical tools to analyze (1).

We assume that there exist $a_{0}>0$ and $p \geq 0$ such that for all $r>0$,

$$
a(r) \geq r\left|a^{\prime}(r)\right|, \quad a(r) \geq \frac{a_{0}}{r^{p}+r^{-p}} .
$$

The first condition means that $a$ grows at most linearly (see Lemma 6). The second condition is a technical assumption needed for the entropy method (see the proof of Lemma 5). Examples are $a(r)=1$, which leads to uncoupled heat equations for $u_{1}$ and $u_{2}, a(r)=r^{\alpha}$ with $0<\alpha \leq 1, a(r)=r^{\beta} /\left(1+r^{\beta-1}\right)$ with $\beta>0$, and $a(r)=1 / r$. The last example gives the equations

$$
\partial_{t} u_{1}=\Delta u_{2}, \quad \partial_{t} u_{2}=\Delta\left(\frac{u_{2}^{2}}{u_{1}}\right)
$$

Surprisingly, this system corresponds (up to a factor) to an energy-transport model for semiconductors. Indeed, introducing the electron density $n:=u_{1}$ and the electron temperature $\theta:=u_{2} / u_{1}$, Eq. (3) can be written as

$$
\partial_{t} n=\Delta(n \theta), \quad \partial_{t}(n \theta)=\Delta\left(n \theta^{2}\right) .
$$

A class of energy-transport models that includes the above example was analyzed in [13].

Another class of models which resembles (1) are the equations

$$
\partial_{t} u_{i}=\Delta\left(p_{i}(u) u_{i}\right), \quad i=1, \ldots, m,
$$

modeling the time evolution of population densities $u_{i}$. These systems are analyzed in, e.g., [5,8], essentially for $m=2$. In this application, $p_{i}$ is often given by the sum $p_{i 1}\left(u_{1}\right)+p_{i 2}\left(u_{2}\right)$, and consequently, the results of $[5,8]$ do not apply and we need to develop new ideas.

Our first main result is the global-in-time existence of weak solutions to (1).

Theorem 1. (Existence of weak solutions) Let (2) hold and let $T>0, \alpha \geq p+4, \mu_{1}, \mu_{2} \in \mathbb{R}, 0 \leq a \in$ $C^{1}(0, \infty), u^{0}=\left(u_{1}^{0}, u_{2}^{0}\right) \in L^{2}\left(\mathbb{T}^{d}\right)^{2}$ with $u_{1}^{0}, u_{2}^{0} \geq 0$ in $\mathbb{T}^{d}$ and $H\left[u^{0}\right]<\infty$. Then, there exists a solution $u=\left(u_{1}, u_{2}\right)$ to (1) satisfying $u_{i}>0$ in $\mathbb{T}^{d}, t>0, i=1,2$, and

$$
\begin{aligned}
& u_{i}, a\left(u_{1} / u_{2}\right) u_{i} \in L^{\infty}\left(0, T ; L^{2}\left(\mathbb{T}^{d}\right)\right), \\
& \quad \nabla u_{i}, \nabla\left(a\left(u_{1} / u_{2}\right) u_{i}\right) \in L^{2}\left(0, T ; L^{2}\left(\mathbb{T}^{d}\right)\right), \quad \partial_{t} u_{i} \in L^{2}\left(0, T ; H^{1}\left(\mathbb{T}^{d}\right)^{\prime}\right), \quad i=1,2 .
\end{aligned}
$$

If additionally $\mu_{i} \leq 0$ for $i=1,2$, we have the uniform bounds

$$
u_{i}, a\left(u_{1} / u_{2}\right) u_{i} \in L^{\infty}\left(0, \infty ; L^{2}\left(\mathbb{T}^{d}\right)\right), \quad \nabla u_{i}, \nabla\left(a\left(u_{1} / u_{2}\right) u_{i}\right) \in L^{2}\left(0, \infty ; L^{2}\left(\mathbb{T}^{d}\right)\right) .
$$

As mentioned above, the proof of this theorem is based on entropy methods. These methods have been originally developed to understand the large-time behavior of solutions; see, e.g., [2,12]. The "entropy" of system (1) is often understood as a convex Lyapunov functional which provides suitable nonlinear gradient estimates. In many situations, and also in the financial context presented here, the "entropy" has no physical counterpart. However, we claim that this notion is appropriate since it naturally generalizes physical situations. For details, we refer to [8].

Our key idea is to employ the functional

$$
H[u]=\int_{\mathbb{T}^{d}} h(u) \mathrm{d} x, \quad h(u)=\left(\frac{u_{1}}{u_{2}}\right)^{\alpha} u_{1}^{2}+\left(\frac{u_{1}}{u_{2}}\right)^{-\alpha} u_{2}^{2}+u_{1}-\log u_{1}+u_{2}-\log u_{2},
$$


where $\alpha \geq p+4$ and $u=\left(u_{1}, u_{2}\right) \in(0, \infty)^{2}$. We will show that

$$
\frac{\mathrm{d}}{\mathrm{d} t} H[u]+\int_{\mathbb{T}^{d}}\left(\left(\frac{u_{1}}{u_{2}}\right)^{\alpha-p}+\left(\frac{u_{1}}{u_{2}}\right)^{p-\alpha}\right)\left(\left|\nabla u_{1}\right|^{2}+\left|\nabla u_{2}\right|^{2}\right) \mathrm{d} x \leq C H[u]
$$

for some constant $C>0$ which vanishes if $\mu_{1}=\mu_{2}=0$. In this situation, the mapping $t \mapsto H[u(t)]$ is nonincreasing; otherwise, for $\mu_{i} \neq 0, t \mapsto H[u(t)]$ is bounded on finite time intervals. We infer from the inequality $x+x^{-1} \geq 2$ for all $x>0$ uniform bounds for $u_{i}(t)$ in $H^{1}\left(\mathbb{T}^{d}\right)$, which are needed for the compactness argument.

The entropy method gives more than just the a priori estimate (7). Indeed, let us write (1) in divergence form:

$$
\partial_{t} u-\operatorname{div}(A(u) \nabla u)=f(u), \quad t>0, \quad u(0)=u^{0} \quad \text { in } \mathbb{T}^{d},
$$

where the $i$ th component of $\operatorname{div}(A(u) \nabla u)$ equals $\sum_{j=1}^{d} \sum_{k=1}^{2} \partial_{j}\left(A_{i k}(u) \partial_{j} u_{k}\right), \partial_{j}=\partial / \partial x_{j}$, and $f(u)=$ $\left(\mu_{1} u_{1}, \mu_{2} u_{2}\right)^{\top}$. The diffusion matrix

$$
A(u)=\left(\begin{array}{cc}
a\left(u_{1} / u_{2}\right)+\left(u_{1} / u_{2}\right) a^{\prime}\left(u_{1} / u_{2}\right) & -\left(u_{1} / u_{2}\right)^{2} a^{\prime}\left(u_{1} / u_{2}\right) \\
a^{\prime}\left(u_{1} / u_{2}\right) & a\left(u_{1} / u_{2}\right)-\left(u_{1} / u_{2}\right) a^{\prime}\left(u_{1} / u_{2}\right)
\end{array}\right)
$$

is generally neither symmetric nor positive definite. Since the only eigenvalue of $A(u)$ is given by $\lambda=$ $a\left(u_{1} / u_{2}\right)>0$, the system is normally elliptic [1], and local-in-time existence of classical solutions can be expected. The difficulty is to prove the global-in-time existence. The entropy density $h(u)$ allows us to formulate (1) in new variables with a positive semidefinite diffusion matrix. Then, together with the a priori estimates from (7), global existence will be deduced. Indeed, defining the so-called entropy variable $w=\left(w_{1}, w_{2}\right)$ by $w_{i}=\partial h / \partial u_{i}(i=1,2)$, Eq. (1) is equivalent to

$$
\partial_{t} u-\operatorname{div}(B(w) \nabla w)=f(u), \quad t>0, \quad u(0)=u^{0} \quad \text { in } \mathbb{T}^{d},
$$

where $B(w)=A(u) h^{\prime \prime}(u)^{-1}$ is positive semidefinite (see Lemma 5 ) and $h^{\prime \prime}(u)$ is the Hessian matrix of $h(u)$. With this formulation, we obtain

$$
\frac{\mathrm{d}}{\mathrm{d} t} H[u]+\int_{\mathbb{T}^{d}} \nabla u: h^{\prime \prime}(u) A(u) \nabla u \mathrm{~d} x=\int_{\mathbb{T}^{d}} f(u) \cdot w \mathrm{~d} x,
$$

where $A: B=\sum_{j=1}^{d} \sum_{k=1}^{2} A_{k j} B_{k j}$ for two matrices $A=\left(A_{k j}\right), B=\left(B_{k j}\right) \in \mathbb{R}^{2 \times d}$. The right-hand side can be bounded in terms of $H[u]$ [see (14)], and the integral on the left-hand side is related to the corresponding integral in (7).

The proof of Theorem 1 is based on a regularization of (9), the fixed-point theorem of Leray-Schauder, and the de-regularization limit. The compactness is obtained from the entropy estimate (7). This technique is similar to those employed in our works $[8,13]$. The novelty here is the (nontrivial) observation that the cross-diffusion system (1) possesses a convex Lyapunov functional, defined by (6). Moreover, compared to $[8,13]$, we are facing additional technical difficulties due to the quotient $u_{1} / u_{2}$.

The second result concerns the large-time asymptotics in the case $\mu_{i}=0$ for $i=1,2$.

Theorem 2. (Large-time asymptotics) Let the assumptions of Theorem 1 hold and let $\mu_{1}=\mu_{2}=0$. Then, the solution $u(t)=\left(u_{1}, u_{2}\right)(t)$ to $(1)$ converges in $L^{2}\left(\mathbb{T}^{d}\right)$ to $\bar{u}=\left(\bar{u}_{1}, \bar{u}_{2}\right)$ as $t \rightarrow \infty$, where

$$
\bar{u}_{i}=\frac{1}{\operatorname{meas}\left(\mathbb{T}^{d}\right)} \int_{\mathbb{T}^{d}} u_{i}^{0} d x, \quad i=1,2 .
$$

If $\mu_{i}<0$ for $i=1,2$, we prove the exponential convergence of $u(t)$ to zero in $H^{1}\left(\mathbb{T}^{d}\right)^{\prime}$, see Remark 9 . For a discussion of the case $\mu_{i}>0$, we refer to Remark 10 . 
The paper is organized as follows. In Sect. 2, we make precise the derivation of (1) from a FokkerPlanck equation. Some technical results are proved in Sect. 3. Section 4 is devoted to the proof of Theorem 1 , and Theorem 2 is shown in Sect. 5.

\section{Derivation of the cross-diffusion system (1)}

We summarize the formal derivation of (1) from a Fokker-Planck equation as presented by Lions [7]. Consider the $n$-dimensional Itō process $X_{t}=\left(X_{t}^{1}, \ldots, X_{t}^{n}\right)$ on some probability space, driven by the $n$-dimensional Wiener process $W_{t}=\left(W_{t}^{1}, \ldots, W_{t}^{n}\right)$ with respect to some given filtration. We assume that $X_{t}$ solves the stochastic differential equation

$$
d X_{t}=\widetilde{\mu}_{t}\left(X_{t}\right) \mathrm{d} t+\sigma_{t}\left(X_{t}\right) d W_{t}, \quad t>0
$$

where $\widetilde{\mu}_{t}=\left(\widetilde{\mu}_{t}^{1}, \ldots, \widetilde{\mu}_{t}^{n}\right)$, and $\sigma_{t}=\left(\sigma_{t}^{i j}\right)_{i, j,=1, \ldots, n}$ is an $n \times n$ matrix. It is well known [10, Theorems 7.3.3, 8.2.1] that the probability density $f\left(x_{1}, \ldots, x_{n}, t\right)$ for $X_{t}$ satisfies the Fokker-Planck (or forward Kolmogorov) equation

$$
\partial_{t} f=\frac{1}{2} \sum_{i, j=1}^{n} \frac{\partial^{2}}{\partial x_{i} \partial x_{j}}\left(D_{i j}(\widehat{x}) f\right)-\sum_{i=1}^{n} \frac{\partial}{\partial x_{i}}\left(\widetilde{\mu}^{i}(\widehat{x}) f\right), \quad \widehat{x} \in \mathbb{R}^{n}, \quad t>0,
$$

where $D(\widehat{x})=\left(D_{i j}(\widehat{x})\right)=\sigma(\widehat{x}) \sigma(\widehat{x})^{\top}$ is the diffusion tensor and $\widehat{x}=\left(x_{1}, \ldots, x_{n}\right)$.

In the following, we set $\widetilde{\mu}_{t}=0$ and $\sigma_{t}=\operatorname{diag}\left(\sigma_{1}, \ldots, \sigma_{n}\right)$. This means that we neglect correlations between the processes. Taking them into account will lead to first-order terms in the final equations; see Remark 3. Under the above simplifications, the Fokker-Planck equation becomes

$$
\partial_{t} f=\frac{1}{2} \sum_{j=1}^{n} \frac{\partial^{2}}{\partial x_{j}^{2}}\left(\sigma_{j}^{2} f\right), \quad \widehat{x} \in \mathbb{R}^{n}, \quad t>0 .
$$

We assume that $\sigma_{j}$ is a function of the partial averages

$$
u_{i}(x, t)=\int_{\mathbb{R}} f\left(x, x_{n}, t\right) e^{\lambda_{i} x_{n}} \mathrm{~d} x_{n}, \quad x=\left(x_{1}, \ldots, x_{n-1}\right), \quad i=1, \ldots, m,
$$

where $\lambda_{i}$ are some given (pairwise different) parameters. Temporal averages appear, for instance, in the modeling of Asian options. Here, $u_{i}$ may be interpreted as an average with respect to the ecocnomic parameter $x_{n}$. We may employ other weights than the exponential one, but this one is mathematically extremely convenient because of the property $\partial u_{i} / \partial x_{n}=\lambda_{i} u_{i}$ (see Remark 3). Multiplying (10) by $e^{\lambda_{i} x_{n}}$ and integrating with respect to $x_{n} \in \mathbb{R}$, a straightforward calculation shows that $u_{i}$ solves

$$
\partial_{t} u_{i}=\frac{1}{2} \sum_{j=1}^{n-1} \frac{\partial^{2}}{\partial x_{j}^{2}}\left(\sigma_{j}^{2} u_{i}\right)+\frac{\lambda_{i}^{2}}{2} \sigma_{n}^{2} u_{i}, \quad i=1, \ldots, m .
$$

We allow $\sigma_{j}$ to depend on the partial averages, $\sigma_{j}=\sigma_{j}\left(u_{1}, \ldots, u_{m}\right)$.

We consider only the special case $m=2, \sigma:=\sigma_{j}$ for $j=1, \ldots, n-1$, and $\sigma_{n}$ is constant and positive. Setting $u=\left(u_{1}, u_{2}\right), \mu_{i}:=\lambda_{i}^{2} \sigma_{n} / 2$, we find that

$$
\partial_{t} u_{i}=\frac{1}{2} \Delta\left(\sigma(u)^{2} u_{i}\right)+\mu_{i} u_{i}, \quad x \in \mathbb{R}^{n-1}, \quad t>0, \quad i=1,2 .
$$

In divergence form, this system is equivalent to

$$
\partial_{t} u=\operatorname{div}(A(u) \nabla u), \quad \text { where } A(u)=\sigma\left(\begin{array}{cc}
\sigma+2 \partial_{1} \sigma u_{1} & 2 \partial_{2} \sigma u_{1} \\
2 \partial_{1} \sigma u_{2} & \sigma+2 \partial_{2} \sigma u_{2}
\end{array}\right),
$$

where $\partial_{i} \sigma=\partial \sigma / \partial u_{i}, i=1,2$. This system is of parabolic type in the sense of Petrovski if the real parts of the eigenvalues of $A$ are nonnegative [1], i.e., if $\sigma+\partial_{1} \sigma u_{1}+\partial_{2} \sigma u_{2} \geq 0$ for all $u \in \mathbb{R}^{2}$. This requirement 
is fulfilled if, for instance, $\sigma$ depends on the quotient $u_{1} / u_{2}$ only. Therefore, we set $\sigma(u)^{2}=2 a\left(u_{1} / u_{2}\right)$. Then,

$$
\partial_{t} u_{i}=\Delta\left(a\left(u_{1} / u_{2}\right) u_{i}\right)+\mu_{i} u_{i}, \quad x \in \mathbb{R}^{n-1}, \quad t>0, \quad i=1,2,
$$

is of parabolic type in the sense of Petrovski, and these equations correspond to (1).

Remark 3. (Generalizations) The general model for nonvanishing $\widetilde{\mu}_{t}^{i}$ and nondiagonal $\sigma_{t}$ is derived as above, and the result reads as

$$
\partial_{t} u_{i}=\frac{1}{2} \sum_{j, k=1}^{n-1} \frac{\partial^{2}}{\partial x_{j} \partial x_{k}}\left(D_{j k} u_{i}\right)-\frac{1}{2} \sum_{j=1}^{n-1} \frac{\partial}{\partial x_{j}}\left(\left(2 \widetilde{\mu}^{j}+\lambda_{i} D_{j n}\right) u_{i}\right)+\frac{\lambda_{i}}{2}\left(2 \widetilde{\mu}^{n}+\lambda_{i} D_{n n}\right) u_{i} .
$$

Compared to (11), this equation also contains first-order terms. If $\widetilde{\mu}_{t}^{i}=0$ and $\sigma_{t}$ is diagonal, we obtain $m$ equations of the type (12). The analysis of cross-diffusion systems with more than two components is expected to be much more involved than for those with two components. For instance, the analysis of the cross-diffusion model (4) is rather well understood only in the case of $m=2$ components, while the case of $m \geq 3$ equations requires additional properties [3].

Another generalization concerns nonexponential weights. For instance, we may define

$$
u_{i}=\int_{\mathbb{R}} f(x, t) \sin \left(\lambda_{i} x_{n}\right) \mathrm{d} x_{n}, \quad i=1, \ldots, m .
$$

Choosing again $\widetilde{\mu}_{t}^{i}=0$ and $\sigma_{t}=\operatorname{diag}\left(\sigma_{1}, \ldots, \sigma_{n}\right)$, we find that

$$
\partial_{t} u_{i}=\frac{1}{2} \sum_{j=1}^{n-1} \frac{\partial^{2}}{\partial x_{j}^{2}}\left(\sigma_{j}(u)^{2} u_{i}\right)-\frac{\lambda_{i}^{2}}{2} \sigma_{n}^{2} u_{i}, \quad i=1, \ldots, m .
$$

This justifies the assumption $\mu_{i} \in \mathbb{R}$ in (1), but there seems to be no financial interpretation of the trigonometric weight functions.

\section{Some auxiliary lemmas}

In this section, we prove some algebraic properties of the matrices $h^{\prime \prime}(u)$ and $A(u)$ and some estimates related to the entropy density $h(u)$ and the components of $A(u)$. Recall that $h(u)$ is defined in (6) and $A(u)$ in (8).

Lemma 4. (Properties of $h$ ) Let $\alpha>0$. The function $h:(0, \infty)^{2} \rightarrow \mathbb{R}^{2}$, defined in (6), is convex, its derivative $h^{\prime}$ is invertible, and there exists $C_{h}>0$ such that for all $u=\left(u_{1}, u_{2}\right) \in(0, \infty)^{2}$,

$$
h(u) \geq \frac{1}{2}\left(u_{1}^{2}+u_{2}^{2}\right), \quad \sum_{i=1}^{2} \mu_{i} u_{i} \partial_{i} h(u) \leq C_{h} h(u),
$$

where we recall that $\partial_{i} h=\partial h / \partial u_{i}$.

Proof. We proceed in several steps.

Step 1: $h$ is convex We compute the first partial derivatives of $h$,

$$
\begin{aligned}
& \partial_{1} h(u)=(\alpha+2) u_{1}^{\alpha+1} u_{2}^{-\alpha}-\alpha u_{1}^{-\alpha-1} u_{2}^{\alpha+2}-u_{1}^{-1}+1, \\
& \partial_{2} h(u)=(\alpha+2) u_{1}^{-\alpha} u_{2}^{\alpha+1}-\alpha u_{1}^{\alpha+2} u_{2}^{-\alpha-1}-u_{2}^{-1}+1,
\end{aligned}
$$


and the Hessian $h^{\prime \prime}(u)=H^{(1)}+H^{(2)}+H^{(3)}$, where

$$
\begin{aligned}
H^{(1)} & =\left(\begin{array}{cc}
(\alpha+2)(\alpha+1)\left(u_{1} / u_{2}\right)^{\alpha} & -\alpha(\alpha+2)\left(u_{1} / u_{2}\right)^{\alpha+1} \\
-\alpha(\alpha+2)\left(u_{1} / u_{2}\right)^{\alpha+1} & \alpha(\alpha+1)\left(u_{1} / u_{2}\right)^{\alpha+2}
\end{array}\right), \\
H^{(2)} & =\left(\begin{array}{cc}
\alpha(\alpha+1)\left(u_{2} / u_{1}\right)^{\alpha+2} & -\alpha(\alpha+2)\left(u_{2} / u_{1}\right)^{\alpha+1} \\
-\alpha(\alpha+2)\left(u_{2} / u_{1}\right)^{\alpha+1} & (\alpha+2)(\alpha+1)\left(u_{2} / u_{1}\right)^{\alpha}
\end{array}\right), \\
H^{(3)} & =\left(\begin{array}{cc}
u_{1}^{-2} & 0 \\
0 & u_{2}^{-2}
\end{array}\right) .
\end{aligned}
$$

Since $\operatorname{det} H^{(1)}=\alpha(\alpha+2)\left(u_{1} / u_{2}\right)^{2(\alpha+1)}>0, \operatorname{det} H^{(2)}=\alpha(\alpha+2)\left(u_{2} / u_{1}\right)^{2(\alpha+1)}>0$, and the diagonal elements of $H^{(1)}, H^{(2)}$ are positive, the matrices $H^{(i)}, i=1,2,3$, are positive definite and so does $h^{\prime \prime}(u)$. Thus, $h$ is convex.

Step 2: $h^{\prime}$ is invertible Since the Hessian $h^{\prime \prime}$ is positive definite on $(0, \infty)^{2}, h^{\prime}$ is one-to-one and the image $R\left(h^{\prime}\right)$ is open. If $R\left(h^{\prime}\right)$ is also closed, it follows that $R\left(h^{\prime}\right)=\mathbb{R}^{2}$ which means that $h^{\prime}$ is surjective. For this, let $\left(w_{n}\right) \in R\left(h^{\prime}\right)$ for $n \in \mathbb{N}$ such that $w_{n} \rightarrow w$ as $n \rightarrow \infty$. We show that $w \in R\left(h^{\prime}\right)$. By definition, there exists $u_{n}>0$ such that $w_{n}=h^{\prime}\left(u_{n}\right)$ for $n \in \mathbb{N}$. The idea is to prove that $\left(u_{n}\right)=\left(u_{1, n}, u_{2, n}\right)$ is a bounded and strictly positive sequence. This implies that, up to a subsequence, $u_{n} \rightarrow u \in(0, \infty)^{2}$ as $n \rightarrow \infty$. By continuity of $h^{\prime}$, we infer that $h^{\prime}\left(u_{n}\right) \rightarrow h^{\prime}(u)$ as $n \rightarrow \infty$. We already know that $h^{\prime}\left(u_{n}\right)=w_{n} \rightarrow w$ which shows that $w=h^{\prime}(u) \in R\left(h^{\prime}\right)$, and $R\left(h^{\prime}\right)$ is closed.

It remains to verify that there exist positive constants $m, M>0$ such that $m \leq u_{i, n} \leq M$ for all $n \in \mathbb{N}, i=1,2$. We argue by contradiction. Let us assume that (up to a subsequence) $u_{1, n} \rightarrow 0$ as $n \rightarrow \infty$. Since $\left(w_{1, n}\right)=\left(\partial_{1} h\left(u_{n}\right)\right)$ is convergent, we deduce from (15) that $u_{2, n} \rightarrow 0$ as well. As a consequence,

$$
\alpha u_{1, n} w_{1, n}+(\alpha+2) u_{2, n} w_{2, n} \rightarrow 0, \quad(\alpha+2) u_{1, n} w_{1, n}+\alpha u_{2, n} w_{2, n} \rightarrow 0 .
$$

Expanding these expressions yields

$$
u_{1, n}^{-\alpha} u_{2, n}^{\alpha+2} \rightarrow \frac{1}{2}, \quad u_{1, n}^{\alpha+2} u_{2, n}^{-\alpha} \rightarrow \frac{1}{2},
$$

and the product also converges, $u_{1, n}^{2} u_{2, n}^{2} \rightarrow 1 / 4$. This is absurd since $\left(u_{n}\right)$ converges to zero. Therefore, $u_{1, n}$ is strictly positive. With an analogous argument, we conclude that $u_{2, n}$ is strictly positive too.

Let us assume that (up to a subsequence) $u_{1, n} \rightarrow \infty$ as $n \rightarrow \infty$. Again, the convergence of $\left(w_{1, n}\right)$ and (15) imply that $u_{2, n} \rightarrow \infty$. Consequently,

$$
\frac{\alpha}{u_{2, n}} w_{1, n}+\frac{\alpha+2}{u_{1, n}} w_{2, n} \rightarrow 0, \quad \frac{\alpha+2}{u_{2, n}} w_{1, n}+\frac{\alpha}{u_{1, n}} w_{2, n} \rightarrow 0
$$

from which we infer after expanding these expressions that $u_{2, n} / u_{1, n} \rightarrow 0$ and $u_{1, n} / u_{2, n} \rightarrow 0$, which is a contradiction. So, $\left(u_{1, n}\right)$ is bounded, and the same conclusion holds for $\left(u_{2, n}\right)$.

Step 3: proof of (14) Observing that $x-\log x \geq 1$ for all $x>0$, it follows that

$$
h(u) \geq u_{1}^{2}\left(\left(\frac{u_{1}}{u_{2}}\right)^{\alpha}+\left(\frac{u_{2}}{u_{1}}\right)^{\alpha+2}\right)=u_{2}^{2}\left(\left(\frac{u_{1}}{u_{2}}\right)^{\alpha+2}+\left(\frac{u_{2}}{u_{1}}\right)^{\alpha}\right) .
$$

The elementary inequality $x^{\alpha}+(1 / x)^{\alpha+2} \geq 1$ for $x>0$ shows the first inequality in (14):

$$
h(u) \geq \frac{u_{1}^{2}}{2}\left(\left(\frac{u_{1}}{u_{2}}\right)^{\alpha}+\left(\frac{u_{2}}{u_{1}}\right)^{\alpha+2}\right)+\frac{u_{2}^{2}}{2}\left(\left(\frac{u_{1}}{u_{2}}\right)^{\alpha+2}+\left(\frac{u_{2}}{u_{1}}\right)^{\alpha}\right) \geq \frac{1}{2}\left(u_{1}^{2}+u_{2}^{2}\right) .
$$


For the second inequality in (14), we employ definition (6) of $h$ and the elementary inequality $x-1 \leq$ $2(x-\log x)$ for $x>0$ to find that, if $C_{h}=2(\alpha+2)\left(\left|\mu_{1}\right|+\left|\mu_{2}\right|\right)$,

$$
\begin{aligned}
\sum_{i=1}^{2} \mu_{i} u_{i} \partial_{i} h(u)= & \left(\mu_{1}(\alpha+2)-\mu_{2} \alpha\right) u_{1}^{\alpha+2} u_{2}^{-\alpha}+\left(\mu_{2}(\alpha+2)-\mu_{1} \alpha\right) u_{1}^{-\alpha} u_{2}^{\alpha+2} \\
& +\mu_{1}\left(u_{1}-1\right)+\mu_{2}\left(u_{2}-1\right) \\
\leq & C_{h}\left(u_{1}^{\alpha+2} u_{2}^{-\alpha}+u_{1}^{-\alpha} u_{2}^{\alpha+2}\right)+C_{h}\left(u_{1}-\log u_{1}+u_{2}-\log u_{2}\right) .
\end{aligned}
$$

This finishes the proof.

Next, we prove that $\left.h^{\prime \prime}(u) A\right)(u)$ is positive semidefinite. Then, $B=A(u) h^{\prime \prime}(u)^{-1}$ in $(9)$ is positive semidefinite too, since $z^{\top} A(u) h^{\prime \prime}(u)^{-1} z=\left(h^{\prime \prime}(u)^{-1} z\right)^{\top} h^{\prime \prime}(u) A(u)\left(h^{\prime \prime}(u)^{-1} z\right) \geq 0$ for $z \in \mathbb{R}^{2}$.

Lemma 5. (Positive semidefiniteness of $h^{\prime \prime} A$ ) Let condition (2) hold. If $\alpha(\alpha+2)>1$, the matrix $h^{\prime \prime}(u) A(u)$ is positive semidefinite in $(0, \infty)^{2}$. Furthermore, if additionally $\alpha \geq p$, there exists a constant $\kappa=\kappa(\alpha)>0$ such that for all $u=\left(u_{1}, u_{2}\right) \in(0, \infty)^{2}$ and $z \in \mathbb{R}^{2}$,

$$
z^{\top} h^{\prime \prime}(u) A(u) z \geq \kappa\left(\left(\frac{u_{1}}{u_{2}}\right)^{\alpha-p}+\left(\frac{u_{1}}{u_{2}}\right)^{p-\alpha}\right)|z|^{2} .
$$

Proof. Let $\alpha(\alpha+2)>1$ and let $M^{(i)}=\left(M_{j k}^{(i)}\right):=\frac{1}{2}\left(\left(H^{(i)} A\right)^{\top}+H^{(i)} A\right)$ be the symmetric part of $H^{(i)} A$, where $H^{(i)}$ with $i=1,2,3$ is defined in (17). A computation shows that

$$
\begin{aligned}
M_{11}^{(1)} & =(\alpha+2)\left((\alpha+1) a\left(u_{1} / u_{2}\right)+\left(u_{1} / u_{2}\right) a^{\prime}\left(u_{1} / u_{2}\right)\right)\left(u_{1} / u_{2}\right)^{\alpha}, \\
\operatorname{det} M^{(1)} & =\left(\alpha(\alpha+2) a\left(u_{1} / u_{2}\right)^{2}-\left(u_{1} / u_{2}\right)^{2} a^{\prime}\left(u_{1} / u_{2}\right)^{2}\right)\left(u_{1} / u_{2}\right)^{2 \alpha+2}, \\
M_{11}^{(2)} & =\alpha\left((\alpha+1) a\left(u_{1} / u_{2}\right)-\left(u_{1} / u_{2}\right) a^{\prime}\left(u_{1} / u_{2}\right)\right)\left(u_{2} / u_{1}\right)^{\alpha+2}, \\
\operatorname{det} M^{(2)} & =\left(\alpha(\alpha+2) a\left(u_{1} / u_{2}\right)^{2}-\left(u_{1} / u_{2}\right)^{2} a^{\prime}\left(u_{1} / u_{2}\right)^{2}\right)\left(u_{2} / u_{1}\right)^{2 \alpha+2}, \\
M^{(3)} & =\left(\begin{array}{cc}
\left(a\left(u_{1} / u_{2}\right)+\left(u / u_{2}\right) a^{\prime}\left(u_{1} / u_{2}\right)\right) u_{1}^{-2} & 0 \\
0 & \left(a\left(u_{1} / u_{2}\right)-\left(u_{1} / u_{2}\right) a^{\prime}\left(u_{1} / u_{2}\right)\right) u_{2}^{-2}
\end{array}\right) .
\end{aligned}
$$

By the first condition in (2) and the positivity of $\alpha$, we infer that $M^{(3)}$ is positive semidefinite and $M_{11}^{(1)}, M_{11}^{(2)}$ are positive for $u, v>0$. Moreover, since $\alpha(\alpha+2)>1$ by assumption, $\operatorname{det}\left(M^{(1)}\right)>0$ and $\operatorname{det}\left(M^{(2)}\right)>0$. Thus, by Sylvester's criterion, $\left(h^{\prime \prime} A\right)(u)$ is positive semidefinite for all $u \in(0, \infty)^{2}$.

Now let additionally $\alpha \geq p$. Then, the first condition in (2) shows that

$$
\begin{aligned}
\frac{\operatorname{det} M^{(1)}}{\operatorname{tr} M^{(1)}} & =\frac{\alpha(\alpha+2) a\left(u_{1} / u_{2}\right)^{2}-\left(u_{1} / u_{2}\right)^{2} a^{\prime}\left(u_{1} / u_{2}\right)^{2}}{\left(\alpha\left(u_{1} / u_{2}\right)^{2}+\alpha+2\right)\left((\alpha+1) a\left(u_{1} / u_{2}\right)+\left(u_{1} / u_{2}\right) a^{\prime}\left(u_{1} / u_{2}\right)\right)}\left(u_{1} / u_{2}\right)^{\alpha+2} \\
& \geq \frac{(\alpha(\alpha+2)-1) a\left(u_{1} / u_{2}\right)^{2}}{(\alpha+2)\left(\left(u_{1} / u_{2}\right)^{2}+1\right)(\alpha+2) a\left(u_{1} / u_{2}\right)}\left(u_{1} / u_{2}\right)^{\alpha+2} \\
& =k_{1}(\alpha) \frac{a\left(u_{1} / u_{2}\right)}{\left(u_{1} / u_{2}\right)^{2}+1}\left(u_{1} / u_{2}\right)^{\alpha+2}
\end{aligned}
$$

where $k(\alpha)=(\alpha(\alpha+2)-1) /(\alpha+2)^{2}$. In a similar way, we find that

$$
\begin{aligned}
\frac{\operatorname{det} M^{(2)}}{\operatorname{tr} M^{(2)}} & =\frac{\alpha(\alpha+2) a\left(u_{1} / u_{2}\right)^{2}-\left(u_{1} / u_{2}\right)^{2} a^{\prime}\left(u_{1} / u_{2}\right)^{2}}{\left((\alpha+2)\left(u_{1} / u_{2}\right)^{2}+\alpha\right)\left((\alpha+1) a\left(u_{1} / u_{2}\right)-\left(u_{1} / u_{2}\right) a^{\prime}\left(u_{1} / u_{2}\right)\right)}\left(u_{2} / u_{1}\right)^{\alpha} \\
& \geq \frac{(\alpha(\alpha+2)-1) a\left(u_{1} / u_{2}\right)^{2}}{(\alpha+2)\left(\left(u_{1} / u_{2}\right)^{2}+1\right)(\alpha+2) a\left(u_{1} / u_{2}\right)}\left(u_{1} / u_{2}\right)^{-\alpha} \\
& =k(\alpha) \frac{a\left(u_{1} / u_{2}\right)}{\left(u_{1} / u_{2}\right)^{2}+1}\left(u_{1} / u_{2}\right)^{-\alpha} .
\end{aligned}
$$


Since $\operatorname{det} M / \operatorname{tr} M$ is a lower bound for the eigenvalues of any symmetric positive definite matrix $M \in \mathbb{R}^{2 \times 2}$ (and taking into account that $M^{(3)}$ is positive definite), we deduce that for $z \in \mathbb{R}^{2}$,

$$
\begin{aligned}
z^{\top}\left(h^{\prime \prime} A\right)(u) z & \geq k(\alpha) a\left(u_{1} / u_{2}\right) \frac{\left(u_{1} / u_{2}\right)^{\alpha+2}+\left(u_{1} / u_{2}\right)^{-\alpha}}{\left(u_{1} / u_{2}\right)^{2}+1}|z|^{2} \\
& \geq \frac{1}{2} k(\alpha) a\left(u_{1} / u_{2}\right)\left(\left(u_{1} / u_{2}\right)^{\alpha}+\left(u_{1} / u_{2}\right)^{-\alpha}\right)|z|^{2} .
\end{aligned}
$$

In the last inequality, we have employed the elementary inequality $\left(x^{\alpha+2}+x^{-\alpha}\right) /\left(x^{2}+1\right) \geq \frac{1}{2}\left(x^{\alpha}+x^{-\alpha}\right)$ which is equivalent to $\left(x^{2}-1\right)\left(x^{\alpha}-x^{-\alpha}\right) \geq 0$, and this holds true for all $x>0$. By the second condition in $(2)$,

$$
z^{\top}\left(h^{\prime \prime} A\right)(u) z \geq \frac{a_{0}}{2} k(\alpha) \frac{\left(u_{1} / u_{2}\right)^{\alpha}+\left(u_{1} / u_{2}\right)^{-\alpha}}{\left(u_{1} / u_{2}\right)^{p}+\left(u_{1} / u_{2}\right)^{-p}}|z|^{2} .
$$

The inequality $\left(x^{\alpha}+x^{-\alpha}\right) /\left(x^{p}+x^{-p}\right) \geq \frac{1}{2}\left(x^{\alpha-p}+x^{p-\alpha}\right)$ is equivalent to $\left(x^{\alpha-p}-x^{p-\alpha}\right)\left(x^{p}-x^{-p}\right) \geq 0$, which holds true for $x>0$ since $\alpha-p \geq 0$ and $p \geq 0$. Therefore,

$$
\left.z^{\top}\left(h^{\prime \prime} A\right)(u) z \geq \frac{a_{0}}{4} k(\alpha)\left(\left(u_{1} / u_{2}\right)^{\alpha-p}+\left(u_{1} / u_{2}\right)^{p-\alpha}\right)\right)|z|^{2},
$$

which concludes the proof with $\kappa=a_{0} k(\alpha) / 4$.

The following two lemmas concern elementary estimates for $a(r)$.

Lemma 6. Given $r_{0}>0$ arbitrary, it holds that

$$
a(r) \leq \begin{cases}\frac{a\left(r_{0}\right)}{r_{0}} r & \text { for } r \geq r_{0} \\ r_{0} a\left(r_{0}\right) \frac{1}{r} & \text { for } r<r_{0}\end{cases}
$$

Proof. The first inequality in (2) implies that $r \mapsto a(r) / r$ is nonincreasing, while $r \mapsto a(r) r$ is nondecreasing. Writing these monotonicity properties in an explicit way gives the result.

Lemma 7. Let $\alpha \geq 2$. Then, for all $u_{1}, u_{2}>0$,

$$
\begin{aligned}
a\left(\frac{u_{1}}{u_{2}}\right)^{2}\left(u_{1}^{2}+u_{2}^{2}\right) & \leq C_{a}\left(u_{1}^{2}+u_{2}^{2}+\frac{u_{1}^{4}}{u_{2}^{2}}\right) \\
& \leq \xi_{\alpha} C_{a}\left(\left(\frac{u_{1}}{u_{2}}\right)^{\alpha} u_{1}^{2}+\left(\frac{u_{1}}{u_{2}}\right)^{-\alpha} u_{2}^{2}\right) \leq \xi_{\alpha} C_{a} h(u),
\end{aligned}
$$

where $C_{a}=a(1)^{2}$ and $\xi_{\alpha}>0$ is a suitable constant which only depends on $\alpha$.

Proof. The first inequality follows from an application of Lemma 6 with $r_{0}=1$. Indeed, if $u_{1} / u_{2} \geq 1$, we obtain

$$
a\left(\frac{u_{1}}{u_{2}}\right)^{2} u_{1}^{2} \leq a(1)^{2} \frac{u_{1}^{2}}{u_{2}^{2}} u_{1}^{2}, \quad a\left(\frac{u_{1}}{u_{2}}\right)^{2} u_{2}^{2} \leq a(1)^{2} u_{1}^{2},
$$

while if $u_{1} / u_{2} \leq 1$ we have

$$
a\left(\frac{u_{1}}{u_{2}}\right)^{2} u_{1}^{2} \leq a(1)^{2} u_{2}^{2}, \quad a\left(\frac{u_{1}}{u_{2}}\right)^{2} u_{2}^{2} \leq a(1)^{2} \frac{u_{2}^{2}}{u_{1}^{2}} u_{2}^{2} .
$$

These inequalities show the claim with $C_{a}=a(1)^{2}$. The second inequality follows from

$$
u_{1}^{2}+u_{2}^{2}+\frac{u_{1}^{4}}{u_{2}^{2}}+\frac{u_{2}^{4}}{u_{1}^{2}}=u_{1} u_{2}\left(\frac{u_{1}}{u_{2}}+\frac{u_{2}}{u_{1}}+\frac{u_{1}^{3}}{u_{2}^{3}}+\frac{u_{2}^{3}}{u_{1}^{3}}\right) \leq \xi_{\alpha} u_{1} u_{2}\left(\frac{u_{1}^{\alpha+1}}{u_{2}^{\alpha+1}}+\frac{u_{2}^{\alpha+1}}{u_{1}^{\alpha+1}}\right),
$$

where $\xi_{\alpha}>0$ is a suitable constant, which depends only on $\alpha$. This finishes the proof. 
Lemma 8. Recall that $A(u)=\left(A_{i j}(u)\right)$ is given by (8). Then, there exists $C_{A}>0$, only depending on $a(1)$, such that for all $u_{1}, u_{2}>0$,

$$
|A(u)| \leq C_{A}\left(1+\left(\frac{u_{1}}{u_{2}}\right)^{2}+\left(\frac{u_{1}}{u_{2}}\right)^{-2}\right) .
$$

Proof. We apply the first condition in (2) to find that

$$
\sum_{i, j=1}^{2}\left|A_{i j}(u)\right| \leq a\left(\frac{u_{1}}{u_{2}}\right)\left(4+\frac{u_{1}}{u_{2}}+\frac{u_{2}}{u_{1}}\right) .
$$

Then Lemma 6 with $r_{0}=1$ implies that

$$
\sum_{i, j=1}^{2}\left|A_{i j}(u)\right| \leq a(1)\left(\frac{u_{1}}{u_{2}}+\frac{u_{2}}{u_{1}}\right)\left(4+\frac{u_{1}}{u_{2}}+\frac{u_{2}}{u_{1}}\right) .
$$

This estimate and Young's inequality conclude the proof.

\section{Proof of Theorem 1}

Let $T>0, N \in \mathbb{N}, \tau=T / N$, and $m \in \mathbb{N}$ with $m>d / 2$. Then, the embedding $H^{m}\left(\mathbb{T}^{d}\right) \hookrightarrow L^{\infty}\left(\mathbb{T}^{d}\right)$ is compact. Furthermore, let $w^{k-1}=\left(w_{1}^{k-1}, w_{2}^{k-1}\right) \in L^{\infty}\left(\mathbb{T}^{d}\right)^{2}$ be given and let $u^{k-1}=\left(h^{\prime}\right)^{-1}\left(w^{k-1}\right)$. By Lemma 4 , the pair $u^{k-1}=\left(u_{1}^{k-1}, u_{2}^{k-1}\right)$ is well defined and we have $u^{k-1} \in L^{\infty}\left(\mathbb{T}^{d}\right)^{2}$. We wish to find $w_{k}=\left(w_{1}^{k}, w_{2}^{k}\right) \in H^{m}\left(\mathbb{T}^{d}\right)^{2}$ such that for all $\phi=\left(\phi_{1}, \phi_{2}\right) \in H^{m}\left(\mathbb{T}^{d}\right)^{2}$,

$$
\begin{aligned}
& \frac{1}{\tau} \int_{\mathbb{T}^{d}}\left(u^{k}-u^{k-1}\right) \cdot \phi \mathrm{d} x+\int_{\mathbb{T}^{d}} \nabla \phi: B\left(w^{k}\right) \nabla w^{k} \mathrm{~d} x \\
& \quad+\tau \int_{\mathbb{T}^{d}}\left(D^{m} w^{k} \cdot D^{m} \phi+w^{k} \cdot \phi\right) \mathrm{d} x=\sum_{i=1}^{2} \mu_{i} \int_{\mathbb{T}^{d}} u_{i}^{k} \phi_{i} \mathrm{~d} x
\end{aligned}
$$

where $B\left(w^{k}\right)=A\left(u^{k}\right) h^{\prime \prime}\left(u^{k}\right)^{-1}$,

$$
D^{m} w^{k} \cdot D^{m} \phi:=\sum_{|\alpha|=m} \sum_{i=1}^{2} D^{\alpha} u_{i}^{k} D^{\alpha} \phi_{i},
$$

$\alpha=\left(\alpha_{1}, \ldots, \alpha_{d}\right) \in \mathbb{N}_{0}^{d}$ is a multiindex and $D^{\alpha}=\partial^{|\alpha|} /\left(\partial x_{1}^{\alpha_{1}} \cdots \partial x_{d}^{\alpha_{d}}\right)$ a partial derivative of order $|\alpha|$.

Step 1: solution of $(18)$ Let $\widehat{w}=\left(\widehat{w}_{1}, \widehat{w}_{2}\right) \in L^{\infty}\left(\mathbb{T}^{d}\right)^{2}$ and $\eta \in[0,1]$ be given. Set $\widehat{u}=\left(\widehat{u}_{1}, \widehat{u}_{2}\right):=$ $\left(h^{\prime}\right)^{-1}(\widehat{w})$. We solve first the linear problem

$$
a(w, \phi)=\eta F(\phi) \quad \text { for all } \phi \in H^{m}\left(\mathbb{T}^{d}\right)^{2},
$$

where

$$
\begin{aligned}
a(w, \phi) & =\int_{\mathbb{T}^{d}}\left(D^{m} w^{k} \cdot D^{m} \phi+w^{k} \cdot \phi\right) \mathrm{d} x+\int_{\mathbb{T}^{d}} \nabla \phi: B(\widehat{w}) \nabla w^{k} \mathrm{~d} x, \\
F(\phi) & =-\frac{1}{\tau} \int_{\mathbb{T}^{d}}\left(\widehat{u}-u^{k-1}\right) \cdot \phi \mathrm{d} x+\sum_{i=1}^{2} \mu_{i} \int_{\mathbb{T}^{d}} \widehat{u}_{i}^{k} \phi_{i} \mathrm{~d} x .
\end{aligned}
$$

Since $\widehat{w} \in L^{\infty}\left(\mathbb{T}^{d}\right)^{2}$ and $h^{\prime}$ is continuous in $(0, \infty)^{2}$, we have $\widehat{u} \in L^{\infty}\left(\mathbb{T}^{d}\right)^{2}$. This shows that $F$ is continuous on $H^{m}\left(\mathbb{T}^{d}\right)$. The bilinear form $a$ is continuous and coercive, by the generalized Poincaré inequality for $H^{m}$ spaces [11, Chap. 2.1.4, Formula (1.39)] and the positive semidefiniteness of $B(\widehat{w})$ (see Lemma 5). 
Hence, the Lax-Milgram lemma provides a unique solution $w=\left(w_{1}, w_{2}\right) \in H^{m}\left(\mathbb{T}^{d}\right)^{2} \hookrightarrow L^{\infty}\left(\mathbb{T}^{d}\right)^{2}$ to $(19)$. This defines the fixed-point operator $S: L^{\infty}\left(\mathbb{T}^{d}\right)^{2} \times[0,1] \rightarrow L^{\infty}\left(\mathbb{T}^{d}\right)^{2}, S(\widehat{w}, \eta)=w$, where $w$ solves $(19)$.

It holds clearly $S(w, 0)=0$. Standard arguments show that $S$ is continuous (see, e.g., the proof of Lemma 5 in [8]). Because of the compact embedding $H^{m}\left(\mathbb{T}^{d}\right) \hookrightarrow L^{\infty}\left(\mathbb{T}^{d}\right)$, the mapping $S$ is even compact. In order to apply the Leray-Schauder fixed-point theorem, it remains to prove a uniform bound for all fixed points of $S(\cdot, \eta)$ in $L^{\infty}\left(\mathbb{T}^{d}\right)^{2}$.

Let $w \in L^{\infty}\left(\mathbb{T}^{d}\right)^{2}$ be such a fixed point, i.e., a solution to (19) with $\widehat{u}$ replaced by $u:=\left(h^{\prime}\right)^{-1}(w)$. The uniform bound will be a consequence of the entropy inequality. For this, we employ the test function $w$ in (19):

$$
\begin{aligned}
& \frac{\eta}{\tau} \int_{\mathbb{T}^{d}}\left(u-u^{k-1}\right) \cdot w \mathrm{~d} x+\int_{\mathbb{T}^{d}} \nabla w: B(w) \nabla w \mathrm{~d} x+\tau \int_{\mathbb{T}^{d}}\left(\left|D^{m} w\right|^{2}+|w|^{2}\right) \mathrm{d} x \\
& \quad=\eta \sum_{i=1}^{2} \mu_{i} \int_{\mathbb{T}^{d}} u_{i} w_{i} \mathrm{~d} x .
\end{aligned}
$$

By the convexity of $h$, it follows that

$$
h(u)-h\left(u^{k-1}\right) \leq h^{\prime}(u) \cdot\left(u-u^{k-1}\right)=\left(u-u^{k-1}\right) \cdot w .
$$

Moreover, by (9) and Lemma 5, we have

$$
\begin{aligned}
\int_{\mathbb{T}^{d}} \nabla w: B(w) \nabla w \mathrm{~d} x & =\int_{\mathbb{T}^{d}} \nabla u:\left(h^{\prime \prime} A\right)(u) \nabla u \mathrm{~d} x \\
& \geq \kappa \int_{\mathbb{T}^{d}}\left(\left(\frac{u_{1}}{u_{2}}\right)^{\alpha-p}+\left(\frac{u_{1}}{u_{2}}\right)^{p-\alpha}\right)|\nabla u|^{2} \mathrm{~d} x .
\end{aligned}
$$

Taking into account the second estimate in (14), we infer that

$$
\eta \sum_{i=1}^{2} \mu_{i} \int_{\mathbb{T}^{d}} u_{i} w_{i} \mathrm{~d} x=\eta \sum_{i=1}^{2} \mu_{i} \int_{\mathbb{T}^{d}} u_{i} \partial_{i} h(u) \mathrm{d} x \leq C_{h} \int_{\mathbb{T}^{d}} h(u) \mathrm{d} x .
$$

Therefore, (20) becomes

$$
\begin{aligned}
& \frac{\eta}{\tau} \int_{\mathbb{T}^{d}} h(u) \mathrm{d} x+\kappa \int_{\mathbb{T}^{d}}\left(\left(\frac{u_{1}}{u_{2}}\right)^{\alpha-p}+\left(\frac{u_{1}}{u_{2}}\right)^{p-\alpha}\right)|\nabla u|^{2} \mathrm{~d} x \\
& \quad+\tau \int_{\mathbb{T}^{d}}\left(\left|D^{m} w\right|^{2}+|w|^{2}\right) \mathrm{d} x \leq \frac{\eta}{\tau} \int_{\mathbb{T}^{d}} h\left(u^{k-1}\right) \mathrm{d} x+C_{h} \int_{\mathbb{T}^{d}} h(u) \mathrm{d} x .
\end{aligned}
$$

Choosing $\tau<1 / C_{h}$, this shows that $w$ is uniformly bounded in $H^{m}\left(\mathbb{T}^{d}\right)$. Thus, we can apply the fixedpoint theorem of Leray-Schauder to conclude the existence of a weak solution $w^{k}:=w$ with $u^{k}=h^{\prime}\left(w^{k}\right)$ to (18) with $\eta=1$.

Step 2: a priori estimates Inequality (21) shows, for $w=w^{k}, u=u^{k}$, and $\eta=1$, that

$$
\begin{aligned}
& \left(1-C_{h} \tau\right) \int_{\mathbb{T}^{d}} h\left(u^{k}\right) \mathrm{d} x+\kappa \tau \int_{\mathbb{T}^{d}}\left(\left(\frac{u_{1}^{k}}{u_{2}^{k}}\right)^{\alpha-p}+\left(\frac{u_{1}^{k}}{u_{2}^{k}}\right)^{p-\alpha}\right)\left|\nabla u^{k}\right|^{2} \mathrm{~d} x \\
& +\tau^{2} \int_{\mathbb{T}^{d}}\left(\left|D^{m} w^{k}\right|^{2}+\left|w^{k}\right|^{2}\right) \mathrm{d} x \leq \int_{\mathbb{T}^{d}} h\left(u^{k-1}\right) \mathrm{d} x .
\end{aligned}
$$


We sum (21) for $k=1, \ldots, j$ and divide the resulting inequality by $1-C_{h} \tau$ (recall that we have chosen $\left.\tau<1 / C_{h}\right)$ :

$$
\begin{aligned}
\int_{\mathbb{T}^{d}} h\left(u^{j}\right) \mathrm{d} x+\frac{\kappa \tau}{1-C_{h} \tau} \sum_{k=1}^{j} \int_{\mathbb{T}^{d}}\left(\left(\frac{u_{1}^{k}}{u_{2}^{k}}\right)^{\alpha-p}+\left(\frac{u_{1}^{k}}{u_{2}^{k}}\right)^{p-\alpha}\right)\left|\nabla u^{k}\right|^{2} \mathrm{~d} x \\
\quad+\frac{\tau^{2}}{1-C_{h} \tau} \sum_{k=1}^{j} \int_{\mathbb{T}^{d}}\left(\left|D^{m} w^{k}\right|^{2}+\left|w^{k}\right|^{2}\right) \mathrm{d} x \\
\leq \frac{1}{1-C_{h} \tau} \int_{\mathbb{T}^{d}} h\left(u^{0}\right) \mathrm{d} x+\frac{C_{h} \tau}{1-C_{h} \tau} \sum_{k=1}^{j-1} \int_{\mathbb{T}^{d}} h\left(u^{k}\right) \mathrm{d} x .
\end{aligned}
$$

We apply the discrete Gronwall inequality [4] to obtain for $j \tau \leq T$,

$$
\begin{aligned}
& \int_{\mathbb{T}^{d}} h\left(u^{j}\right) \mathrm{d} x+\tau \sum_{k=1}^{j} \int_{\mathbb{T}^{d}}\left(\left(\frac{u_{1}^{k}}{u_{2}^{k}}\right)^{\alpha-p}+\left(\frac{u_{1}^{k}}{u_{2}^{k}}\right)^{p-\alpha}\right)\left|\nabla u^{k}\right|^{2} \mathrm{~d} x \\
& +\tau^{2} \sum_{k=1}^{j} \int_{\mathbb{T}^{d}}\left(\left|D^{m} w^{k}\right|^{2}+\left|w^{k}\right|^{2}\right) \mathrm{d} x \leq C,
\end{aligned}
$$

where $C>0$ denotes a constant which is independent of $\tau$ (and independent of $T$ if $\mu_{i} \leq 0$ ) but dependent on the initial entropy $H\left[u^{0}\right]$.

We define the piecewise constant functions in time $w^{(\tau)}(x, t)=w^{k}(x)$ and $u^{(\tau)}(x, t)=u^{k}(x)$ for $x \in \mathbb{T}^{d}$ and $t \in((k-1) \tau, k \tau], k=1, \ldots, j$. Furthermore, we introduce the shift operator $\sigma_{\tau} u^{(\tau)}(x, t)=u^{k-1}(x)$ for $x \in \mathbb{T}^{d}, t \in((k-1) \tau, k \tau]$. With this notation, we can rewrite $(20)$ (with $\eta=1$ ) as

$$
\begin{aligned}
& \frac{1}{\tau} \int_{0}^{T} \int_{\mathbb{T}^{d}}\left(u^{(\tau)}-\sigma_{\tau} u^{(\tau)}\right) \cdot \phi \mathrm{d} x \mathrm{~d} t+\int_{0}^{T} \int_{\mathbb{T}^{d}} \nabla \phi:\left(h^{\prime \prime} A\right)\left(u^{(\tau)}\right) \nabla u^{(\tau)} \mathrm{d} x \mathrm{~d} t \\
& \quad+\tau \int_{0}^{T} \int_{\mathbb{T}^{d}}\left(D^{m} w^{(\tau)} \cdot D^{m} \phi+w^{(\tau)} \cdot \phi^{(\tau)}\right) \mathrm{d} x \mathrm{~d} t+\sum_{i=1}^{2} \mu_{i} \int_{0}^{T} \int_{\mathbb{T}^{d}} u_{i}^{(\tau)} \phi_{i} \mathrm{~d} x \mathrm{~d} t
\end{aligned}
$$

and (22) as

$$
\begin{aligned}
& \int_{\mathbb{T}^{d}} h\left(u^{(\tau)}(t)\right) \mathrm{d} x+\int_{0}^{t} \int_{\mathbb{T}^{d}}\left(\left(\frac{u_{1}^{(\tau)}}{u_{2}^{(\tau)}}\right)^{\alpha-p}+\left(\frac{u_{1}^{(\tau)}}{u_{2}^{(\tau)}}\right)^{p-\alpha}\right)\left|\nabla u^{(\tau)}\right|^{2} \mathrm{~d} x \mathrm{~d} s \\
& +\tau \int_{0}^{t} \int_{\mathbb{T}^{d}}\left(\left|D^{m} w^{(\tau)}\right|^{2}+\left|w^{(\tau)}\right|^{2}\right) \mathrm{d} x \mathrm{~d} s \leq C,
\end{aligned}
$$

where $t \in((j-1) \tau, j \tau]$. It follows that

$$
\left\|w^{(\tau)}\right\|_{L^{2}\left(0, T ; H^{m}\left(\mathbb{T}^{d}\right)\right)} \leq C \tau^{-1 / 2}
$$


By Lemmas 7, 4, and estimate (24), we find that

$$
\begin{aligned}
& \int_{\mathbb{T}^{d}}\left(\left|a\left(\frac{u_{1}^{(\tau)}}{u_{2}^{(\tau)}}\right) u_{1}^{(\tau)}\right|^{2}+\left|a\left(\frac{u_{1}^{(\tau)}}{u_{2}^{(\tau)}}\right) u_{2}^{(\tau)}\right|^{2}\right) \mathrm{d} x \leq 3 C_{a} \int_{\mathbb{T}^{d}} h\left(u^{(\tau)}\right) \mathrm{d} x \leq C, \\
& \int_{\mathbb{T}^{d}}\left(\left(u_{1}^{(\tau)}\right)^{2}+\left(u_{2}^{(\tau)}\right)^{2}\right) \mathrm{d} x \leq \int_{\mathbb{T}^{d}} h\left(u^{(\tau)}\right) \mathrm{d} x \leq C .
\end{aligned}
$$

Moreover, using Lemmas 8 and (24),

$$
\begin{gathered}
\int_{0}^{T} \int_{\mathbb{T}^{d}}\left(\left|\nabla\left(a\left(u_{1}^{(\tau)} / u_{2}^{(\tau)}\right) u_{1}^{(\tau)}\right)\right|^{2}+\left|\nabla\left(a\left(u_{1}^{(\tau)} / u_{2}^{(\tau)}\right) u_{2}^{(\tau)}\right)\right|^{2}\right) \mathrm{d} x \mathrm{~d} t \\
\quad=\int_{\mathbb{T}^{d}}\left|A\left(u^{(\tau)}\right) \nabla u^{(\tau)}\right|^{2} \mathrm{~d} x \leq \int_{0}^{T} \int_{\mathbb{T}^{d}}\left|A\left(u^{(\tau)}\right)\right|^{2}\left|\nabla u^{(\tau)}\right|^{2} \mathrm{~d} x \mathrm{~d} t \\
\leq C_{A} \int_{0}^{T} \int_{\mathbb{T}^{d}}\left(1+\left(\frac{u_{1}^{(\tau)}}{u_{2}^{(\tau)}}\right)^{4}+\left(\frac{u_{2}^{(\tau)}}{u_{1}^{(\tau)}}\right)^{4}\right)\left|\nabla u^{(\tau)}\right|^{2} \mathrm{~d} x \mathrm{~d} t \\
\leq C \int_{0}^{T} \int_{\mathbb{T}^{d}}\left(\left(\frac{u_{1}^{(\tau)}}{u_{2}^{(\tau)}}\right)^{\alpha-p}+\left(\frac{u_{1}^{(\tau)}}{u_{2}^{(\tau)}}\right)^{p-\alpha}\right)\left|\nabla u^{(\tau)}\right|^{2} \mathrm{~d} x \mathrm{~d} t \leq C .
\end{gathered}
$$

The last but one inequality follows from the elementary estimate $1+y^{4} \leq y^{\alpha-p}+y^{p-\alpha}$ for $y>0$ which holds because of the assumption $\alpha-p \geq 4$. Estimates (26)-(28) yield for $i=1,2$,

$$
\begin{gathered}
\left\|u_{i}^{(\tau)}\right\|_{L^{\infty}\left(0, T ; L^{2}\left(\mathbb{T}^{d}\right)\right)}+\left\|\nabla u_{i}^{(\tau)}\right\|_{L^{2}\left(0, T ; L^{2}\left(\mathbb{T}^{d}\right)\right)} \leq C, \\
\left\|a\left(u_{1}^{(\tau)} / u_{2}^{(\tau)}\right) u_{i}\right\|_{L^{\infty}\left(0, T ; L^{2}\left(\mathbb{T}^{d}\right)\right)}+\left\|\nabla\left(a\left(u_{1}^{(\tau)} / u_{2}^{(\tau)}\right) u_{i}^{(\tau)}\right)\right\|_{L^{2}\left(0, T ; L^{2}\left(\mathbb{T}^{d}\right)\right)} \leq C .
\end{gathered}
$$

These estimates are uniform in $T>0$ if $\mu_{i} \leq 0$.

Next, we derive a uniform estimate for the discrete time derivative $\left(u^{(\tau)}-\sigma_{\tau} u^{(\tau)}\right) / \tau$. For $\phi \in$ $L^{2}\left(0, T ; H^{m}\left(\mathbb{T}^{d}\right)\right)$, we estimate

$$
\begin{aligned}
\frac{1}{\tau}\left|\int_{0}^{T}\left(u^{(\tau)}-\sigma_{\tau} u^{(\tau)}\right) \cdot \phi \mathrm{d} x \mathrm{~d} t\right| \leq & \left\|A\left(u^{(\tau)}\right) \nabla u^{(\tau)}\right\|_{L^{2}\left(0, T ; L^{2}\left(\mathbb{T}^{d}\right)\right)}\|\nabla \phi\|_{L^{2}\left(0, T ; L^{2}\left(\mathbb{T}^{d}\right)\right)} \\
& +\tau\left\|w^{(\tau)}\right\|_{L^{2}\left(0, T ; H^{m}\left(\mathbb{T}^{d}\right)\right)}\|\phi\|_{L^{2}\left(0, T ; H^{m}\left(\mathbb{T}^{d}\right)\right)} \\
& +\max \left\{\mu_{1}, \mu_{2}\right\}\left\|u^{(\tau)}\right\|_{L^{2}\left(0, T ; L^{2}\left(\mathbb{T}^{d}\right)\right)}\|\phi\|_{L^{2}\left(0, T ; L^{2}\left(\mathbb{T}^{d}\right)\right)} \\
\leq & C\|\phi\|_{L^{2}\left(0, T ; H^{m}\left(\mathbb{T}^{d}\right)\right)}
\end{aligned}
$$

taking into account the bounds (25), (28), and (29). Therefore,

$$
\tau^{-1}\left\|u^{(\tau)}-\sigma_{\tau} u^{(\tau)}\right\|_{L^{2}\left(0, T ; H^{m}\left(\mathbb{T}^{d}\right)^{\prime}\right)} \leq C .
$$

Step 3: limit $\tau \rightarrow 0$ Estimates (29) and (31) allow us to apply the Aubin-Lions lemma in the discrete version of [6] to obtain the existence of a subsequence, which is not relabeled, such that, as $\tau \rightarrow 0$,

$$
u_{i}^{(\tau)} \rightarrow u_{i} \quad \text { strongly in } L^{2}\left(0, T ; L^{2}\left(\mathbb{T}^{d}\right)\right) \text { and a.e., } \quad i=1,2 .
$$


Moreover, by (25), (29), and (31), for the same subsequence and $i=1,2$,

$$
\begin{aligned}
\tau w_{i}^{(\tau)} \rightarrow 0 & \text { strongly in } L^{2}\left(0, T ; H^{m}\left(\mathbb{T}^{d}\right)\right), \\
\nabla u_{i}^{(\tau)} \rightarrow \nabla u_{i} & \text { weakly in } L^{2}\left(0, T ; L^{2}\left(\mathbb{T}^{d}\right)\right), \\
\tau^{-1}\left(u_{i}^{(\tau)}-\sigma_{\tau} u_{i}^{(\tau)}\right) \rightarrow \partial_{t} u_{i} & \text { weakly in } L^{2}\left(0, T ; H^{m}\left(\mathbb{T}^{d}\right)^{\prime}\right) .
\end{aligned}
$$

The pointwise convergence of $\left(u_{i}^{(\tau)}\right)$, Fatou's lemma, and estimate $(24)$ imply that, for a.e. $t \in(0, T)$,

$$
\begin{aligned}
\sum_{i=1}^{2} \int_{\mathbb{T}^{d}}\left(u_{i}(t)-\log u_{i}(t)\right) \mathrm{d} x & \leq \liminf _{\tau \rightarrow 0} \sum_{i=1}^{2} \int_{\mathbb{T}^{d}}\left(u_{i}^{(\tau)}(t)-\log u_{i}^{(\tau)}(t)\right) \mathrm{d} x \\
& \leq \liminf _{\tau \rightarrow 0} \int_{\mathbb{T}^{d}} h\left(u^{(\tau)}(t)\right) \mathrm{d} x \leq C .
\end{aligned}
$$

This means that $u_{i}>0$ a.e. in $\mathbb{T}^{d} \times(0, T)$.

Estimate (26) and (28) show that, up to a subsequence,

$$
a\left(u_{1}^{(\tau)} / u_{2}^{(\tau)}\right) u_{i}^{(\tau)} \rightarrow q_{i} \quad \text { weakly in } L^{2}\left(0, T ; H^{1}\left(\mathbb{T}^{d}\right)\right), i=1,2,
$$

where $q_{i} \in L^{2}\left(0, T ; H^{1}\left(\mathbb{T}^{d}\right)\right)$. We wish to identify $q_{i}$. To this end, let us define $\chi_{\varepsilon}^{(\tau)}=\mathbf{1}_{\left\{u_{1}^{(\tau)} \geq \varepsilon, u_{2}^{(\tau)} \geq \varepsilon\right\}}$ and $\chi_{\varepsilon}=\mathbf{1}_{\left\{u_{1} \geq \varepsilon, u_{2} \geq \varepsilon\right\}}$, where $\mathbf{1}_{A}$ denotes the characteristic function on the set $A$. Clearly, $\chi_{\varepsilon}^{(\tau)} \rightarrow \chi_{\varepsilon}$ strongly in $L^{s}\left(0, T ; L^{s}\left(\mathbb{T}^{d}\right)\right)$ for all $1 \leq s<\infty$. We infer that

$$
\chi_{\varepsilon}^{(\tau)} a\left(u_{1}^{(\tau)} / u_{2}^{(\tau)}\right) u_{i}^{(\tau)} \rightarrow \chi_{\varepsilon} a\left(u_{1} / u_{2}\right) u_{i} \quad \text { weakly in } L^{s}\left(0, T ; L^{s}\left(\mathbb{T}^{d}\right)\right), \quad 1 \leq s<2 .
$$

We deduce that $q_{i}=a\left(u_{1} / u_{2}\right) u_{i}$ on the set $\left\{u_{1} \geq \varepsilon, u_{2} \geq \varepsilon\right\}$. Since $\varepsilon>0$ is arbitrary and $u_{i}>0$ a.e. in $\mathbb{T}^{d} \times(0, T)$, this identification holds, in fact, a.e. in $\mathbb{T}^{d} \times(0, T)$.

Consequently, we may perform the limit $\tau \rightarrow 0$ in (23) to deduce that $u$ is a weak solution to (1) with test functions $L^{2}\left(0, T ; H^{m}\left(\mathbb{T}^{d}\right)^{\prime}\right)$. However, since $a\left(u_{1} / u_{2}\right) u_{i} \in L^{2}\left(0, T ; H^{1}\left(\mathbb{T}^{d}\right)\right)$, we employ a density argument to infer that (1) also holds for $L^{2}\left(0, T ; H^{1}\left(\mathbb{T}^{d}\right)^{\prime}\right)$. Since $u_{i} \in L^{2}\left(0, T ; H^{1}\left(\mathbb{T}^{d}\right)\right)$ and $\partial_{t} u_{i} \in L^{2}\left(0, T ; H^{1}\left(\mathbb{T}^{d}\right)^{\prime}\right)$, it follows that $u_{i} \in C^{0}\left([0, T] ; L^{2}\left(\mathbb{T}^{d}\right)\right)$, so the initial datum is satisfied in $L^{2}\left(\mathbb{T}^{d}\right)$. Finally, since the bounds are uniform in $T$ if $\mu_{i} \leq 0$, the statement (5) follows.

\section{Proof of Theorem 2}

Theorem 1 allows us to employ the test equations $u_{1}-\bar{u}_{1}, u_{2}-\bar{u}_{2}$ in (1), respectively:

$$
\frac{\mathrm{d}}{\mathrm{d} t} \int_{\mathbb{T}^{d}} \sum_{i=1}^{2}\left(u_{i}-\bar{u}_{i}\right)^{2} \mathrm{~d} x=-\int_{\mathbb{T}^{d}} \sum_{i=1}^{2} \nabla u_{i} \cdot \nabla\left(a(u) u_{i}\right) \mathrm{d} x
$$

which, together with Theorem 1 , implies that $(\mathrm{d} / \mathrm{d} t) \int \sum_{i=1}^{2}\left(u_{i}-\bar{u}_{i}\right)^{2} \mathrm{~d} x \in L^{1}(0, \infty)$. Consequently, the limit

$$
\lim _{t \rightarrow \infty} \int_{\mathbb{T}^{d}} \sum_{i=1}^{2}\left(u_{i}(t)-\bar{u}_{i}\right)^{2} \mathrm{~d} x=\int_{\mathbb{T}^{d}} \sum_{i=1}^{2}\left(u_{i}^{0}-\bar{u}_{i}\right)^{2} \mathrm{~d} x+\int_{0}^{\infty} \frac{\mathrm{d}}{\mathrm{d} t} \int_{\mathbb{T}^{d}} \sum_{i=1}^{2}\left(u_{i}-\bar{u}_{i}\right)^{2} \mathrm{~d} x \mathrm{~d} t
$$

exists and is finite. Poincaré's inequality and Theorem 1 imply that

$$
\int_{\mathbb{T}^{d}} \sum_{i=1}^{2}\left(u_{i}-\bar{u}_{i}\right)^{2} \mathrm{~d} x \leq C_{P} \int_{\mathbb{T}^{d}} \sum_{i=1}^{2}\left|\nabla u_{i}\right|^{2} \mathrm{~d} x \in L^{1}(0, \infty),
$$


which means that $\lim _{t \rightarrow \infty} \int_{\mathbb{T}^{d}} \sum_{i=1}^{2}\left(u_{i}(t)-\bar{u}_{i}\right)^{2} \mathrm{~d} x=0$. This finishes the proof.

Remark 9. If $\mu_{i}<0$ for $i=1,2$, we can prove the exponential convergence of the solution $u(t)$ to (1) in $H^{1}\left(\mathbb{T}^{d}\right)^{\prime}$ by using the dual method. Indeed, let $\phi_{i} \in L^{2}\left(0, T ; H^{1}\left(\mathbb{T}^{d}\right)\right)$ be the unique solution to $-\Delta \phi_{i}=u_{i}(t)$ in $\mathbb{T}^{d}$ and $\int_{\mathbb{T}^{d}} \phi_{i} \mathrm{~d} x=0, i=1,2$. Employing $\phi=\left(\phi_{1}, \phi_{2}\right)$ as a test function in (1), we find after a straightforward computation that

$$
\frac{1}{2} \frac{\mathrm{d}}{\mathrm{d} t} \int_{\mathbb{T}^{d}}\left(\left|\nabla \phi_{1}\right|^{2}+\left|\nabla \phi_{2}\right|^{2}\right) \mathrm{d} x+\int_{\mathbb{T}^{d}} a\left(u_{1} / u_{2}\right)\left(u_{1}^{2}+u_{2}^{2}\right) \mathrm{d} x=\int_{\mathbb{T}^{d}}\left(\mu_{1}\left|\nabla \phi_{1}\right|^{2}+\mu_{2}\left|\nabla \phi_{2}\right|^{2}\right) \mathrm{d} x .
$$

Then Gronwall's lemma implies that

$$
\int_{\mathbb{T}^{d}}|\nabla \phi(t)|^{2} \mathrm{~d} x \leq e^{\max \left\{\mu_{1}, \mu_{2}\right\} t} \int_{\mathbb{T}^{d}}|\nabla \phi(0)|^{2} \mathrm{~d} x, \quad t>0 .
$$

Since $\left\|u_{i}\right\|_{H^{1}\left(\mathbb{T}^{d}\right)^{\prime}}=\left\|\phi_{i}\right\|_{H^{1}\left(\mathbb{T}^{d}\right)}$, we conclude that $\left\|u_{i}(t)\right\|_{H^{1}\left(\mathbb{T}^{d}\right)^{\prime}} \leq C \exp (-\kappa t)$ for $t>0$, where $\kappa=$ $-\max \left\{\mu_{1}, \mu_{2}\right\}>0$ and $C>0$ depends on $u^{0}$.

Remark 10. In the case $\mu_{i}>0$ for $i=1,2$, we cannot expect equilibration rates, since the solution grows in the $L^{2}$ norm as $t \rightarrow \infty$. This growth can be made precise if $\mu:=\mu_{1}=\mu_{1}>0$. Indeed, $u_{i}^{*}=e^{-\mu t} u_{i}$ solves

$$
\partial_{t} u_{i}^{*}=\Delta\left(a\left(u_{1}^{*} / u_{2}^{*}\right) u_{i}^{*}\right), \quad t>0, \quad u_{i}^{*}(0)=u_{i}^{0} \quad \text { in } \mathbb{T}^{d}, \quad i=1,2,
$$

and Theorem 2 shows that $u_{i}^{*}(t) \rightarrow \bar{u}_{i}$ in $L^{2}\left(\mathbb{T}^{d}\right)$ as $t \rightarrow \infty$, which translates to $\left\|e^{-\mu t} u_{i}(t)-\bar{u}_{i}\right\|_{L^{2}\left(\mathbb{T}^{d}\right)} \rightarrow$ 0 .

\section{Acknowledgements}

Open access funding provided by Austrian Science Fund (FWF).

Open Access. This article is distributed under the terms of the Creative Commons Attribution 4.0 International License (http://creativecommons.org/licenses/by/4.0/), which permits unrestricted use, distribution, and reproduction in any medi$\mathrm{um}$, provided you give appropriate credit to the original author(s) and the source, provide a link to the Creative Commons license, and indicate if changes were made.

\section{References}

[1] Amann, H.: Nonhomogeneous linear and quasilinear elliptic and parabolic boundary value problems. In: Schmeisser, H.J., Triebel, H. (eds.) Function Spaces, Differential Operators and Nonlinear Analysis, p. 9126. Teubner, Stuttgart (1993)

[2] Arnold, A., Markowich, P., Toscani, G., Unterreiter, A.: On convex Sobolev inequalities and the rate of convergence to equilibrium for Fokker-Planck type equations. Commun. Part. Differ. Eqs. 26, 43-100 (2001)

[3] Chen, X., Daus, E., Jüngel, A.: Global existence analysis of cross-diffusion population systems for multiple species. Submitted for publication (2016). arXiv:1608.03696

[4] Clark, D.: Short proof of a discrete Gronwall inequality. Discrete Appl. Math. 16, 279-281 (1987)

[5] Desvillettes, L., Lepoutre, T., Moussa, A., Trescases, A.: On the entropic structure of reaction-cross diffusion systems. Commun. Part. Differ. Eqs. 40, 1705-1747 (2015)

[6] Dreher, M., Jüngel, A.: Compact families of piecewise constant functions in $L^{p}(0, T ; B)$. Nonlinear Anal. 75, 3072-3077 (2012)

[7] Lions, P.-L.: Some new classes of nonlinear Kolmogorov equations. Talk at the 16th Pauli Colloquium, Wolfgang-Pauli Institute, Vienna, November 18 (2015)

[8] Jüngel, A.: The boundedness-by-entropy method for cross-diffusion systems. Nonlinearity 28, 1963-2001 (2015) 
[9] Menz, S., Latorre, J., Schütte, C., Huisinga, W.: Hybrid stochastic-deterministic solution of the chemical master equation. SIAM Multiscale Model. Simul. 10, 1232-1262 (2012)

[10] Øksendal, B.: Stochastic Differential Equations. Springer, Berlin (2003)

[11] Temam, R.: Infinite-Dimensional Dynamical Systems in Mechanics and Physics, 2nd edn. Springer, New York (1997)

[12] Villani, C.: Optimal Transport. Old and New. Springer, Berlin (2009)

[13] Zamponi, N., Jüngel, A.: Global existence analysis for degenerate energy-transport models for semiconductors. J. Differ. Eqs. 258, 2339-2363 (2015)

Ansgar Jüngel and Nicola Zamponi

Institute for Analysis and Scientific Computing

Vienna University of Technology

Wiedner Hauptstraße 8-10

1040 Vienna

Austria

e-mail: juengel@tuwien.ac.at

Nicola Zamponi

e-mail: nicola.zamponi@tuwien.ac.at

(Received: January 19, 2016; revised: November 8, 2016) 